\title{
Three particles in a finite volume
}

\author{
Akaki Rusetsky* \\ Helmholtz-Institut für Strahlen- und Kernphysik (Theorie) and Bethe Center for Theoretical \\ Physics, Universität Bonn, D-53115 Bonn, Germany \\ E-mail: rusetsky@itkp.uni-bonn.de
}

The volume-dependence of a shallow three-particle bound state in the cubic box with a size $L$ is studied. It is shown that, in the unitary limit, the energy-level shift from the infinite-volume position is given by $\Delta E=c\left(\kappa^{2} / m\right)(\kappa L)^{-3 / 2}|A|^{2} \exp (-2 \kappa L / \sqrt{3})$, where $\kappa$ is the bound-state momentum and $|A|^{2}$ denotes the three-body analog of the asymptotic normalization constant, which encodes the information about the short-range interactions in the three-body system.

The 33rd International Symposium on Lattice Field Theory

14 - 18 July 2015

Kobe International Conference Center, Kobe, Japan

*Speaker. 


\section{Introduction}

Despite the fact that there have been several attempts to formulate the Lüscher-type formalism in case of three particles [1-5], the problem is still far from its solution. Conceptually, the central question here is, whether the finite-volume spectrum is determined solely by the observables in the system of three particles in the infinite volume. This statement was proved first in Ref. [1] and subsequently confirmed ${ }^{1}$ in Refs. [2-4]. However, the three-particle quantization condition, given in Refs. [2,3], has still a very complicated form. Hence, even though the problem is solved in principle, a practical use of this solution can still require a major effort. On the other hand, such a formalism is urgently needed to analyze already existing data on the resonances that may decay into the three-particle final states, e.g., the Roper resonance [7] or the $a_{1}(1260)$ meson [8], as well as to correctly interpret the data on the nuclei obtained from lattice QCD and nuclear lattice simulations [9-11]. Since a large amount of far more precise lattice data is expected to appear in the near future, an approach that can be handled with a relative ease, is urgently needed.

The aim of the present work is to study the energy spectrum of the shallow bound states of the three identical non-relativistic bosons in a finite volume. Additionally, it is assumed that the two-body scattering length $a$ is very large, i.e., one is working in the so-called unitary limit. Such a choice is justified by the fact that, for this system, the energy shift from the infinite-volume value can be evaluated analytically in a rather straightforward manner. Moreover, such systems can be studied by using numerical methods (see, e.g., [6]), so one can directly verify the analytic results. In our opinion, the study of the simple systems is interesting, first and foremost, because this gives an insight, how the general quantization condition for the three-particle sector works in practice. The information, gained in the study of this simple case, can be further used to design a framework that can be applied to more complex systems.

In the present paper, we do not treat the three-body bound state by using the quantization condition derived in $[2,3]$. We rather follow the original derivation in the position space, which can be found in Ref. [12] (see also [5, 13], where the formula for the energy shift is generalized to the case of an arbitrary angular momentum). The advantage of such a choice is that the role of the hyperspherical coordinates becomes very transparent in the position space. Namely, we shall see that the asymptotic form of the bound-state wave function can be most easily written down in terms of the hyperspherical coordinates that enables one to derive an analytic expression for the energy shift in a straightforward way. Needless to say that the same result should be obtainable on the basis of the three-particle quantization condition [2,3]. Finally, note that the main results of the present work are contained in Ref. [14].

\section{Derivation of the formula}

The Schrödinger equation for three identical particles with the mass $m$ in the infinite volume reads as

$$
\left\{\sum_{i=1}^{3}\left(-\frac{1}{2 m} \nabla_{i}^{2}+V\left(\mathbf{x}_{i}\right)\right)+E_{T}\right\} \psi\left(\mathbf{r}_{1}, \mathbf{r}_{2}, \mathbf{r}_{3}\right)=0,
$$

\footnotetext{
${ }^{1}$ The authors of Ref. [6] have arrived at the same conclusion on the basis of a direct numerical solution of the three-particle equations in a finite volume.
} 
where $\nabla_{i}=\partial / \partial \mathbf{r}_{i}, E_{T}=\kappa^{2} / m$, and $\kappa$ stands for the three-body bound-state momentum. We further define the Jacobi coordinates

$$
\mathbf{x}_{i}=\mathbf{r}_{j}-\mathbf{r}_{k}, \quad \mathbf{y}_{i}=\frac{1}{\sqrt{3}}\left(\mathbf{r}_{j}+\mathbf{r}_{k}-2 \mathbf{r}_{i}\right)
$$

with $(i j k)=(123),(312),(231)^{2}$.

In a finite volume, the potential $V$ is replaced by

$$
V_{L}\left(\mathbf{x}_{i}\right)=\sum_{\mathbf{n} \in \mathbb{Z}^{3}} V\left(\mathbf{x}_{i}+\mathbf{n} L\right),
$$

and the Schrödinger equation takes the form

$$
\left\{\sum_{i=1}^{3}\left(-\frac{1}{2 m} \nabla_{i}^{2}+V_{L}\left(\mathbf{x}_{i}\right)\right)+E_{L}\right\} \psi_{L}\left(\mathbf{r}_{1}, \mathbf{r}_{2}, \mathbf{r}_{3}\right)=0
$$

Next, let us choose the CM frame. Due to the translational invariance, the bound-state wave functions depend on two Jacobi coordinates $\mathbf{x}_{i}, \mathbf{y}_{i}$. The Bose symmetry in addition implies $\psi\left(\mathbf{x}_{i}, \mathbf{y}_{i}\right)=$ $\psi\left(\mathbf{x}_{k}, \mathbf{y}_{k}\right), i, k=1,2,3$,. The wave function $\psi_{L}$ obeys the same relations.

Our aim here is to evaluate the finite-volume shift $\Delta E=E_{T}-E_{L}$. To this end, we define the trial wave function

$$
\psi_{0}=\sum_{\mathbf{n}, \mathbf{m}} \psi\left(\mathbf{x}_{1}-(\mathbf{n}+\mathbf{m}) L, \mathbf{y}_{1}+\frac{1}{\sqrt{3}}(\mathbf{n}-\mathbf{m}) L\right)
$$

Introducing the notation $H_{L}=\sum_{i=1}^{3}\left(-\frac{1}{2 m} \nabla_{i}^{2}+V_{L}\left(\mathbf{x}_{i}\right)\right)$, it can be checked that $\psi_{0}$ obeys the equation $\left(H_{L}+E_{T}\right) \psi_{0}=\eta$, where

$$
\eta=\sum_{\mathbf{n}, \mathbf{m}} \hat{V}_{\mathbf{n m}} \psi\left(\mathbf{x}_{1}-(\mathbf{n}+\mathbf{m}) L, \mathbf{y}_{1}+\frac{1}{\sqrt{3}}(\mathbf{n}-\mathbf{m}) L\right)
$$

and

$$
\hat{V}_{\mathbf{n m}}=\sum_{\mathbf{k} \neq-\mathbf{n}-\mathbf{m}} V\left(\mathbf{x}_{1}+\mathbf{k} L\right)+\sum_{\mathbf{k} \neq \mathbf{n}} V\left(\mathbf{x}_{2}+\mathbf{k} L\right)+\sum_{\mathbf{k} \neq \mathbf{m}} V\left(\mathbf{x}_{3}+\mathbf{k} L\right) .
$$

Since the potential $V(\mathbf{x})$ is short-ranged, $\eta \propto \exp (-$ const $\cdot \kappa L)$.

It can be verified that the energy level shift to all orders in perturbation theory is given by

$$
\Delta E=\frac{\left\langle\psi_{0}|T| \psi_{0}\right\rangle}{\left\langle\psi_{0} \mid \psi_{0}\right\rangle}, \quad T=\left(H_{L}+E_{T}\right)-\left(H_{L}+E_{T}\right) Q G Q\left(H_{L}+E_{T}\right),
$$

where

$$
G=\frac{1}{H_{L}+E_{L}}, \quad Q=\frac{\left|\psi_{0}\right\rangle\left\langle\psi_{0}\right|}{\left\langle\psi_{0} \mid \psi_{0}\right\rangle} .
$$

\footnotetext{
${ }^{2}$ In Eq. (2.2) no three-body force is present. Its inclusion, however, proceeds in a straightforward manner and does not lead to any complication. This statement is especially important, if the problem is treated within the field-theoretical framework. It is well known that the model with pair interactions only is not renormalizable. Therefore, it is perfectly legitimate to ask, what would happen with our finite-volume results in the presence of the three-body force, which is needed in the infinite volume for canceling the ultraviolet divergences.
} 
The leading exponential correction to the energy shift is given by

$$
\Delta E=\frac{\left\langle\eta \mid \psi_{0}\right\rangle}{\left\langle\psi_{0} \mid \psi_{0}\right\rangle}+\cdots
$$

Here, the ellipses stand for higher-order terms.

What remains to be done is to substitute the expression for $\eta$ from Eq. (2.6) into the above expression and to single out the leading exponential contribution at large $L$. Since $V(\mathbf{x})$ is a shortrange potential, it can be readily seen that at least one of the wave functions in the overlap integral should be evaluated at a large value of the hyperradius $R=\frac{1}{\sqrt{2}}\left(\mathbf{x}_{1}^{2}+\mathbf{y}_{1}^{2}\right)^{1 / 2}$. Since the wave functions decrease exponentially with $R$, the leading exponential correction can be obtained by minimizing the sum of two hyperradii in the wave function. Considering all possible terms in Eq. (2.6), one may conclude that the leading contribution is defined by

$$
\Delta E=6 \cdot 2 \cdot 3 \int d^{3} \mathbf{x}_{1} d^{3} \mathbf{y}_{1} \psi\left(\mathbf{x}_{1}, \mathbf{y}_{1}\right) V\left(\mathbf{x}_{1}\right) \psi\left(\mathbf{x}_{1}-\mathbf{e} L, \mathbf{y}_{1}+\frac{1}{\sqrt{3}} \mathbf{e} L\right)+\cdots,
$$

where $\mathbf{e}=(0,0,1)$ denotes a unit vector and the ellipses stand for the exponentially suppressed terms. In this formula, the infinite-volume wave function $\psi$ is normalized to unity. The factor in front of the integral reflects the symmetries: 6 for different orientations of the unit vector e, 2 for different signs in the second argument of the wave function $\mathbf{y}_{1} \pm \frac{1}{\sqrt{3}} \mathbf{e} L$, and 3 for three different pair potentials.

\section{The energy shift}

The leading-order energy shift is given by Eq. (2.11). As mentioned above, in the calculation of the overlap integral it suffices to replace one of the wave functions by its asymptotic tail, which does not depend on the short-range details of the interaction. The wave function takes a universal form in the unitary limit, which in the context of our problem means that the two-body scattering length $a \geq L$. On the other hand, it is assumed that the interaction range vanishes. Under these assumptions, in the configuration space, the wave function can be approximated by the well-known universal expression (see, e.g. [15])

$$
\psi\left(\mathbf{x}_{1}, \mathbf{y}_{1}\right)=A \mathscr{N} R^{-5 / 2} f_{0}(R) \sum_{i=1}^{3} \frac{\sinh \left(s_{0}\left(\pi / 2-\alpha_{i}\right)\right)}{\sin \left(2 \alpha_{i}\right)} \doteq \sum_{i=1}^{3} \phi\left(R, \alpha_{i}\right),
$$

where

$$
f_{0}(R)=R^{1 / 2} K_{i s_{0}}(\sqrt{2} \kappa R)
$$

and $K_{v}(z)$ denotes the Bessel function. Here, $\alpha_{i}=\arctan \left(\left|\mathbf{x}_{i}\right| /\left|\mathbf{y}_{i}\right|\right)$ and the numerical constant $s_{0} \simeq 1.00624$.

Note that the above expression is valid almost everywhere in the configuration space. The exception is given by the configurations, when two out of three particles stay close together as the hyperradius grows to infinity. We are interested, however, in the overlap integral over all configurations. Since the exceptional configurations have zero measure in the limit of the vanishing effective 
range, they will not give contribution to the final result, provided the rest of the integrand is not singular there. This qualitative discussion gives a clear hint, why the finite-volume spectrum is, at the end, determined solely by the infinite-volume observables, even in the presence of the so-called "spectator" diagrams.

Further, the normalization coefficient $\mathscr{N}$ in Eq. (3.1) is chosen so that

$$
\int d^{3} \mathbf{x}_{1} d^{3} \mathbf{y}_{1}\left|\psi\left(\mathbf{x}_{1}, \mathbf{y}_{1}\right)\right|^{2}=|A|^{2} .
$$

The quantity $|A|^{2}$ is a very important characteristic of a bound state since it encodes the information about the short-range dynamics in the system. It could be interpreted as a three-body analog of the asymptotic normalization coefficient for the wave function. Consider, for example, the situation, when the long-range effects dominate and the true wave function coincides with the asymptotic wave function almost everywhere. Since the true wave function is normalized to unity, the quantity $|A|^{2}$ should be very close to one. In the opposite case, when almost the whole wave function is concentrated at small distances, the quantity $|A|^{2}$ should be very small. It is seen that measuring the quantity $|A|^{2}$ on the lattice will allow one to judge about the nature of a bound state, in analogy to the two-body case (see, e.g., [16] and references therein).

After the general discussion, we turn to the evaluation of the overlap integral in Eq. (2.11) by using the explicit wave function from Eq. (3.1). The second wave function in the integral can be written as

$$
\psi\left(\mathbf{x}_{1}-\mathbf{e} L, \mathbf{y}_{1}+\frac{1}{\sqrt{3}} \mathbf{e} L\right)=\sum_{i=1}^{3} \phi\left(R^{\prime}, \alpha_{i}^{\prime}\right) .
$$

As $L \rightarrow \infty$, the quantity $R^{\prime}$ becomes

$$
R^{\prime}=\frac{\left(\left(\mathbf{x}_{1}-\mathbf{e} L\right)^{2}+\left(\mathbf{y}_{1}+\mathbf{e} L / \sqrt{3}\right)^{2}\right)^{1 / 2}}{\sqrt{2}} \rightarrow \sqrt{\frac{2}{3}} L-\frac{\sqrt{3}}{2 \sqrt{2}} \mathbf{e x}_{1}+\frac{1}{2 \sqrt{2}} \mathbf{e y}_{1}+\cdots
$$

whereas the angular variables tend to the following limiting values:

$$
\begin{aligned}
\tan \alpha_{1}^{\prime} & =\frac{\left|\mathbf{x}_{1}-\mathbf{e} L\right|}{\left|\mathbf{y}_{1}+\mathbf{e} L / \sqrt{3}\right|} \rightarrow \sqrt{3}+\cdots, \\
\tan \alpha_{2}^{\prime} & =\frac{\left|\mathbf{x}_{2}+\mathbf{e} L\right|}{\left|\mathbf{y}_{2}+\mathbf{e} L / \sqrt{3}\right|} \rightarrow \sqrt{3}+\cdots, \\
\tan \alpha_{3}^{\prime} & =\frac{\left|\mathbf{x}_{3}\right|}{\left|\mathbf{y}_{3}-2 \mathbf{e} L / \sqrt{3}\right|} \rightarrow \frac{\sqrt{3}}{2} \frac{\left|\mathbf{x}_{3}\right|}{L}+\cdots \\
& =\frac{\sqrt{6} R \sin \alpha_{3}}{2 L}+\cdots .
\end{aligned}
$$

The expansion of the angular part of the second wave function yields

$$
\sum_{i=1}^{3} \frac{\sinh \left(s_{0}\left(\pi / 2-\alpha_{i}^{\prime}\right)\right)}{\sin \left(2 \alpha_{i}^{\prime}\right)} \rightarrow \frac{L}{\sqrt{6} R} \frac{\sinh \left(\pi s_{0} / 2\right)}{\sin \left(\alpha_{3}\right)}+\cdots
$$

Next, using the equation

$$
\psi\left(\mathbf{x}_{1}, \mathbf{y}_{1}\right) V\left(\mathbf{x}_{1}\right)=\left(\frac{1}{m}\left(\frac{\partial^{2}}{\partial \mathbf{x}_{i}^{2}}+\frac{\partial^{2}}{\partial \mathbf{y}_{i}^{2}}\right)-E_{T}\right) \phi\left(R, \alpha_{1}\right)
$$


and the asymptotic expression for the hyperradial wave function

$$
f_{0}\left(\sqrt{\frac{2}{3}} L\right) \rightarrow \sqrt{\frac{\pi}{2}} \exp \left(-\frac{2 \kappa L}{\sqrt{3}}\right) \exp \left(\frac{\sqrt{3} \kappa}{2} \mathbf{e x}_{1}-\frac{\kappa}{2} \mathbf{e y}_{1}\right) \frac{1}{(\sqrt{2} \kappa)^{1 / 2}}+\cdots,
$$

one may evaluate the overlap intehral in Eq. (2.11). The final result looks as follows:

$$
\Delta E=c\left(\kappa^{2} / m\right)(\kappa L)^{-3 / 2}|A|^{2} \exp (-2 \kappa L / \sqrt{3})+\cdots,
$$

where

$$
c \simeq-96.351
$$

is a numerical constant expressed in terms of $s_{0}$, and the ellipses stand for the sub-leading terms ${ }^{3}$ in $L$.

The Eq. (3.10) displays our main result. Measuring the binding energy at different volumes, one may determine the infinite-volume quantities $E_{T}$ and $|A|^{2}$ through the extrapolation procedure.

\section{Conclusions}

The equation (3.10) is an explicit prediction of the volume dependence for a genuine threebody observable. Understanding this result on the basis of the three-body quantization condition [2, 3] would enable one to gain more insight into the structure of three-body scattering equations in a finite volume and would therefore facilitate the formulation of a framework that can be conveniently used to analyze the lattice data on the three-particle systems.

In the future, we plan to move, step by step, away from the approximations, which were used to derive the result given in Eq. (3.10). Namely, we would like to study the effects of a finite scattering length, perform an effective-range expansion in the potential and include the partial-wave mixing.

\section{Acknowledgments}

The authors would like to thank S. Bour, M. Döring, E. Epelbaum, H.-W. Hammer, M. Hansen, M. Jansen, D. Lee and S. Sharpe for useful discussions. This work is partly supported by the EU Integrated Infrastructure Initiative HadronPhysics3 Project under Grant Agreement no. 283286. We also acknowledge the support by the DFG (CRC 16, "Subnuclear Structure of Matter" and CRC 110, "Symmetries and the Emergence of Structure in QCD") This research is supported in part by Volkswagenstiftung under contract no. 86260 .

\section{References}

[1] K. Polejaeva and A. Rusetsky, Eur. Phys. J. A 48, 67 (2012).

[2] M. T. Hansen and S. R. Sharpe, Phys. Rev. D 90 (2014) 11, 116003 [arXiv:1408.5933 [hep-lat]].

\footnotetext{
${ }^{3}$ In difference to the two-particle case, here the sub-leading terms which are down only by powers of $L$, are also present.
} 
[3] M. T. Hansen and S. R. Sharpe, arXiv:1504.04248 [hep-lat].

[4] R. A. Briceno and Z. Davoudi, Phys. Rev. D 87, no. 9, 094507 (2013).

[5] S. Bour et al., Phys. Rev. D 84, 091503 (2011); S. Bour, H.-W. Hammer, D. Lee and U.-G. Meißner, Phys. Rev. C 86, 034003 (2012).

[6] S. Kreuzer and H.-W. Hammer, Phys. Lett. B 673, 260 (2009); Eur. Phys. J. A 43, 229 (2010); Phys. Lett. B 694, 424 (2011).

[7] N. Mathur et al., Phys. Lett. B 605, 137 (2005); H. W. Lin and S. D. Cohen, AIP Conf. Proc. 1432, 305 (2012); D. S. Roberts, W. Kamleh and D. B. Leinweber, arXiv:1304.0325 [hep-lat].

[8] C. B. Lang, L. Leskovec, D. Mohler and S. Prelovsek, JHEP 1404 (2014) 162 [arXiv:1401.2088 [hep-lat]].

[9] S. R. Beane, W. Detmold, K. Orginos and M. J. Savage, Prog. Part. Nucl. Phys. 66, 1 (2011);

S. R. Beane et al., Phys. Rev. D 87, no. 3, 034506 (2013).

[10] T. Yamazaki et al. [PACS-CS Collaboration], Phys. Rev. D 81, 111504 (2010).

[11] E. Epelbaum, H. Krebs, D. Lee and U.-G. Meißner, Phys. Rev. Lett. 106, 192501 (2011); T. A. Lähde et al., Phys. Lett. B 732, 110 (2014).

[12] M. Lüscher, Commun. Math. Phys. 104, 177 (1986).

[13] S. König, D. Lee and H.-W. Hammer, Phys. Rev. Lett. 107, 112001 (2011); Annals Phys. 327 (2012) 1450 .

[14] U.-G. Meißner, G. Ríos and A. Rusetsky, Phys. Rev. Lett. 114 (2015) 9, 091602 [arXiv:1412.4969 [hep-lat]].

[15] For the description of the three-body bound state in the infinite volume, we mainly follow the review: E. Braaten and H.-W. Hammer, Phys. Rept. 428, 259 (2006), see also references therein.

[16] D. Agadjanov, F.-K. Guo, G. Ríos and A. Rusetsky, JHEP 1501 (2015) 118 [arXiv:1411.1859 [hep-lat]]. 